\title{
A MATEMÁTICA PRESENTE NAS CONVERSÕES DE NÚMEROS NAS BARRAS DA ARITMÉTICA DE LOCALIZAÇÃO
}

\author{
THE MATHEMATICS IN THE NUMBERS CONVERSIONS OF THE RODS OF \\ LOCATION ARITHMETIC
}

\author{
Jeniffer Pires de Almeida ${ }^{1}$; Ana Carolina Costa Pereira ${ }^{2}$
}

\begin{abstract}
RESUMO
Recentemente, tornou-se notável o crescimento de pesquisas, na área da Educação, que buscam meios para auxiliar o professor em formação inicial e continuada, facilitando o processo de ensino dentro de sala de aula. Em relação ao ensino de matemática, alguns estudos relatam a respeito da construção de uma interface entre história e o ensino de matemática, que permite uma forma de buscar na História a compreensão dos conceitos e teorias. Uma das formas de construir-se essa interface é através dos instrumentos matemáticos, que por sua vez têm se mostrado grandes potencializadores didáticos. Com base nisso, essa pesquisa busca expor um estudo sobre o instrumento Aritmética Linear, precisamente no processo de conversão de seus números, contido no método de Aritmética de Localização de John Napier. Tal instrumento está compreendido no tratado Rabdologiae publicado em 1617, contudo esse estudo foi desenvolvido com base em uma pesquisa qualitativa de cunho documental a partir da tradução de William Frank Richardson, de 1990, publicada por Rice, González-Velasco e Corrigan em 2017. A partir dessa pesquisa, percebemos que para começar a se apropriar do instrumento, precisamos entender os métodos de conversões dos números manipulados no mesmo. Portanto, reconhecemos alguns conhecimentos matemáticos, como o cálculo das operações, progressões geométricas e o uso de números binários, que podem contribuir para a interface entre história e ensino da Matemática.

Palavras-chave: História da Matemática. Ensino de Matemática. Rabdologiae. John Napier. Aritmética de Localização.
\end{abstract}

\footnotetext{
ABSTRACT

Recently, there has been a notable growth in researches in the field of Education, which seeks ways to assist the teacher in initial and continuing education, facilitating the teaching process within the classroom. In relation to the teaching of mathematics, some studies report on the construction of an interface between history and the teaching of mathematics, which allows a way of seeking in History the understanding of concepts and theories. One of the ways to build this interface is through mathematical instruments, which in turn have proved to be great didactic

${ }^{1}$ Graduanda de Licenciatura em Matemática na Universidade Estadual do Ceará (UECE). Bolsista de Iniciação Científica e Tecnológica da Universidade Estadual do Ceará (UECE), Fortaleza, Ceará, Brasil. Endereço para correspondência: Av. Silas Munguba, 1700, Itaperi, Fortaleza, Ceará, Brasil, CEP: 60.740903. E-mail: jeniffer.almeida@aluno.uece.br.

ORCID iD: https://orcid.org/0000-0001-5532-8933.

${ }^{2}$ Pós-Doutora em Educação Matemática pela Pontifícia Universidade Católica de São Paulo (PUC-SP). Docente do programa de Pós-Graduação em Educação e do curso de Licenciatura em Matemática da Universidade Estadual do Ceará (UECE), Fortaleza, Ceará, Brasil. Endereço para correspondência: Av. Silas Munguba, 1700, Itaperi, Fortaleza, Ceará, Brasil, CEP: 60.740-903. E-mail: carolina.pereira@uece.br. 
enhancers. Based on this, this research seeks to expose a study on the Linear Arithmetic instrument, precisely in the process of converting its numbers, contained in John Napier's Location Arithmetic method. Such an instrument is included in the treatise Rabdologiae published in 1617, however this study was developed based on a qualitative documentary research based on the translation by William Frank Richardson, from 1990, published by Rice, González-Velasco and Corrigan in 2017. A From this research, we realized that in order to start appropriating the instrument, we need to understand the conversion methods of the numbers manipulated in it. Therefore, we recognize some mathematical knowledge, such as the calculation of operations, geometric progressions and the use of binary numbers, which can contribute to the interface between history and the teaching of Mathematics.

Keywords: History of Mathematics. Math Teaching. Rabdologiae. John Napier. Location Arithmetic. 


\title{
Introdução
}

A educação é uma área que vem reiventando-se dia após dia, o que requer uma exigência por qualificação dos profissionais desse setor. Baseado nisso, tornou-se notável o crescimento de pesquisas nesse campo que buscam meios para auxiliar o professor em sua atuação no magistério. Com isso, tem sido desenvolvida uma necessidade de articular os conhecimentos técnicos-ciéntificos e os saberes pedagógicos, de modo que seja desenvolvido no docente as competências e habilidades capazes de refletir de forma eficaz e construtiva no seu ato de ensinar.

Com a Educação Matemática isso não é diferente, de acordo com Albuquerque e Gontijo (2013, p.80):

\begin{abstract}
a didática da matemática revela-se necessária ao longo de todo o processo de formação, para que o licenciando, num movimento dialético entre o conhecimento específico (matemático) e o conhecimento didático, seja capaz de produzir saberes que serão essenciais na organização e execução do trabalho pedagógico, cuja finalidade é ensinar/aprender matemática.
\end{abstract}

Dessa forma, nos últimos anos, os pesquisadores em Educação Matemática têm voltado sua atenção para a formação de professores que ensinam matemática, dentre essas pesquisas realizadas estão as que apresentam uma proposta de articulação entre história da matemática e ensino de matemática, sob históriografias tradicionais e atualizadas.

Conforme Saito e Dias (2013, p.92) uma interface entre a história e o ensino de matemática, na visão atualizada é um "conjunto de ações e produções que provoca a reflexão sobre o processo histórico da construção do conhecimento matemático para elaborar atividades que busquem articular história e ensino de matemática”. Assim dizendo, essa concepão busca na história recursos didáticos que permitam uma compreensão melhor dos conceitos e teorias matemáticas, como afirma Saito (2016, p.4)

\footnotetext{
Muitas dessas propostas, além de fornecer subsídios para compreensão do papel da história no ensino, procuraram pontuar diferentes vertentes didáticas (e também pedagógicas) que são associadas à história da matemática com vistas a propor novos caminhos de abordagem para o ensino e a aprendizagem de matemática.
}

Uma das formas de mobilização de conhecimento que essa articulação apresenta é por meio de um instrumento matemático que é visto como um "articulador dos dois campos (história da matemática e educação matemática)" (PEREIRA; SAITO, 2018, p. 
109). Neles são investigados possíveis conhecimentos matemáticos, tanto em sua construção como na sua manipulação, que possam vir a se tornar potencializadores didáticos, para facilitar o ensino de Matemática.

A Aritmética de Localização é divida em duas partes, sendo uma delas a construção e manipulação de uma placa, denominada como "Tabuleiro de Xadrez" e a outra a elaboração e utilização de uma barra, intitulada "Aritmética Linear", que pode ser vista como a base para o entendimento do método no geral, pois é nela que são realizados os primeiros processos para o cálculo das operações propostas. Esse instrumento se encontra no tratado Rabdologiae Seu Numerationis Per Virgulas Libri Duo, especificamente no livro de Aritmética de Localização, de Jonh Napier (1550-1617), publicado em 1617.

Procurou-se mostrar que, além da própria manipulação do instrumento, existem conhecimentos matemáticos que emergem de sua construção inicial, visto que, o método de Aritmética de Localização já foi elaborado com o instuito de facilitar operações matemáticas, porém suas regras de manipulação também podem ser vistas como potencializadores no ensino.

Assim sendo, esse trabalho busca apresentar um estudo a respeito do conhecimento matemático presente nas conversões dos números realizadas através da barra de Artimética Linear, utilizada no método de Aritmética de Localização de Jonh Napier. Dessa forma, esse estudo irá se deter na construção dessa barra de Aritmética Linear e ao que ela nos propõe quanto à conversão dos números de Napier.

\section{Metodologia}

Quanto a natureza da nosso estudo, ele se caracteriza como uma pesquisa aplicada, buscando "gerar conhecimentos para aplicação prática dirigidos à solução de problemas específicos" (PRODANOV; FREITAS, 2013, p.51). Assim sendo, realizou-se uma pesquisa de cunho qualitativo, buscando analisar e interpretar a Aritmética Linear para que pudéssemos descrever os conhecimentos matemáticos presentes nela.

Logo, no que se refere ao procedimento, elaborou-se uma pesquisa documental e bibliográfica, uma vez que foi realizada uma tradução e análise do tratado Rabdologiae baseado na tradução de William Frank Richardson, de 1990, publicada por Rice, González-Velasco e Corrigan (2017) e consultando livros, artigos e sites que tratam do 
contexto histórico em que o objeto de estudo estava inserido. A respeito da diferença entre esses dois campos, Prodanov e Freitas (2013, p. 55) explicam que,

\begin{abstract}
Enquanto a pesquisa bibliográfica se utiliza fundamentalmente das contribuições de vários autores sobre determinado assunto, a pesquisa documental baseia-se em materiais que não receberam ainda um tratamento analítico ou que podem ser reelaborados de acordo com os objetivos da pesquisa.
\end{abstract}

Desse modo, esta pesquisa busca através da observação documental e bibliográfica, apresentar alguns conhecimentos matemáticos provenientes da construção e manipulação da Aritmética Linear contida no método de Aritmética de Localização de John Napier, como uma forma de incorporar a história da matemática na educação básica e/ou formação de professores.

\title{
A Artimética Linear
}

A Aritmética de Localização é um artifício desenvolvido por Jonh Napier (15501617) para facilitar o cálculo das operações matemáticas, visto pelo autor como um método "mais fácil e preciso do que qualquer outro método curto de aritmética"3 (NAPIER, 2017, p.727, tradução nossa).

Esse procedimento "é realizado por meio de peças dispostas de acordo com certas regras ao longo de uma linha ou dentro de um quadrado"4 (NAPIER, 2017, p. 728, tradução nossa). Assim dizendo, esse método pode ser desenvolvido de duas formas, na qual a primeira é realizada utilizando um quadrado, que Napier denomina como “Tabuleiro de Xadrez" e a segunda utiliza uma linha, ou uma barra, nomeada de “Artimética Linear", a qual nosso estudo irá deter-se.

A Artimética Linear é uma parte importante do método de Localização de Napier, pois, posteriormente, ela servirá de base para a construção do Tabuleiro de Xadrez, nela que são realizados as operações de adição e subtração, além de efetuar os primeiros processos para a multiplicação, subtração e extração de raízes.

Para a construção dessa haste é preciso primeiramente dividí-la em tantas partes iguais forem necessárias, de acordo com o tamanho do número que deseja-se operar, ou

\footnotetext{
3 "Is easier and more accurate than almost any other short method of Arithmetic" (NAPIER, 2017, p. 727)

4 "Is performed by means of counters set out according to certain rules either along a line or within a square" (NAPIER, 2017, p.728)
} 
seja, o autor não específica um tamanho padrão para essa haste, podendo ela variar de acordo com a preferência e necessidade de quem irá manuseá-la.

No entanto, para realizar essa divisão é importante entender como se dá a disposição dos números nessa haste, sobre isso Napier (2017, p.728, tradução nossa) fala que "são atribuídos valores por um processo contínuo de duplicação" „5, isto é, cada seção irá conter um número que será o dobro do valor anterior, começando pelo número 1.

Além do mais, "todas as seções da haste procedendo para cima na ordem númerica usual são denotadas pelas letras em ordem alfabética"6 (NAPIER, 2017, p.728, tradução nossa), melhor dizendo, cada divisão, denomida como posição, é representada por uma letra, que corresponderá ao número indicado nela, assim, a primeira posição de uma haste de Artimética Linear é indicada pela letra $a$ que será equivalente ao número 1. Exemplificando, se quisermos uma barra com 10 divisões ela irá conter as letras de $a$ a $k$ que representarão os números de 1 a 512, que crescerão de forma que cada um seja o dobro do anterior (Figura 1).

Figura 1 - Barra de Artimética Linear com 10 divisões.

\begin{tabular}{|c|c|}
\hline $\mathbf{k}$ & 512 \\
\hline $\mathbf{i}$ & 256 \\
\hline $\mathbf{h}$ & 128 \\
\hline $\mathbf{g}$ & 64 \\
\hline $\mathbf{f}$ & 32 \\
\hline $\mathbf{e}$ & 16 \\
\hline $\mathbf{d}$ & 8 \\
\hline $\mathbf{c}$ & 4 \\
\hline $\mathbf{b}$ & 2 \\
\hline $\mathbf{a}$ & 1 \\
\hline
\end{tabular}

Fonte: Elaborado pelas autoras.

É importante ressaltar que, cada barra de Aritmética Linear produzida poderá operar com todos os números menores que o dobro do último valor inscrito. Em outras

5 "Are assigned values by a continual process of doubling" (NAPIER, 2017, p.728)

6 "All the sections of the rod proceeding upward in the usual numerical order are denoted by the letters of the alphabet in order" (NAPIER, 2017, p.728) 
palavras, no caso da haste que construímos (Figura 1), o último valor gravado é o 512, então essa barra calculará todos os números menores que 1024, que é o dobro de 512.

Napier (2017) destaca que a letra evidenciada por cada número na Artimética Linear é a notação local daquele devido valor, por exemplo, se quisermos a representação local do número 16, basta olharmos na barra e saberemos que a sua letra correspondente será $e$, mas caso não tenhamos uma representação direta de determinado número, tem-se que usar os métodos de conversão descritas na seção a seguir.

\section{Conversão de números comuns para números locais}

Segundo Napier (2017, p.729, tradução nossa), a conversão de um número comum para um número local pode ser realizada de dois modos, "por subtração ou divisão por dois" ". A partir disso, iremos expressar a respeito desses métodos e exemplificá-los, de modo a facilitar a compreensão.

a) Em relação ao processo de subtração, Almeida e Pereira (2020, p.45) explicam que

\footnotetext{
Consiste em escolher a partir do número fornecido, um número na lateral do tabuleiro que seja mais próximo, porém menor que o primeiro, e, ao realizar a subtração entre eles, executar o mesmo processo com o restante, até que o número fornecido seja totalmente usado, sempre colocando uma peça em cada uma das posições encontradas pelo processo.
}

Ou seja, ao realizar um procedimento contínuo de subtrações com um determinado número comum, a partir dos números dispostos na barra, podemos reescrevê-lo em notação local observando as letras que estão atribuídas a cada uma das posições encontradas na nossa haste de Aritmética Linear no processo de conversão. Para exemplificar, será convertido o número 20 em número local.

\footnotetext{
7 “By subtraction or by division by two" (NAPIER, 2017, p.729)
} 
Figura 2 - Aritmética Linear na representação do método de conversão por subtração.

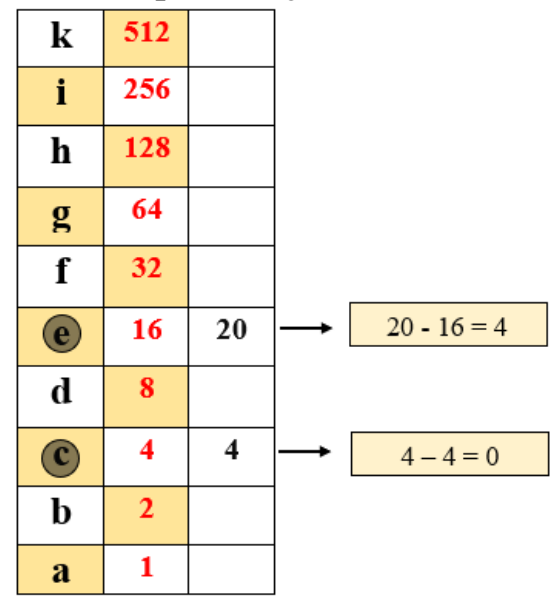

Fonte: Elaborado pelas autoras.

O primeiro passo é encontrar na nossa barra o valor mais próximo, porém menor que o número 20, a saber 16, e realizar a subtração entre eles, resultando em 4, que já encontra-se de forma direta na haste. Ao finalizar o processo e tendo demarcado com peças as posições equivalentes aos números, basta observar as letras correspondentes a eles para escrever o nosso número em notação local, no caso o número 20 será escrito como ec, de acordo com a Figura 2.

b) A respeito do outro método de conversão de um número comum para um número local, que utiliza a divisão por dois, Napier (2017, p.729, tradução nossa) esclarece que

Se o número fornecido for ímpar, coloque um contador na posiçao $a$, subtraia
um e divida o restante por dois. Caso contrário, se o número fornecido for par,
nenhum contador deve ser colocado na posição $a$. Sempre dividindo por dois
e se o resultado for ímpar, subtraindo um e colocando uma peça naquele local,
mas se o resultado for par, não coloque nada, até que o número dado seja
reduzido à unidade, no qual uma peça é colocada na posição alcançada. ${ }^{8}$

Assim dizendo, no método de divisão por dois precisa-se realizar dois processos, de acordo com a paridade do número dado e os quocientes encontrados nas divisões. Caso o número seja ímpar devemos subtrair uma unidade, de modo a encontrar um número divisível por dois, ao fazer isso, temos que demarcar a posição referente à ordem do número subtraído. Por exemplo, se o próprio número dado "X" for ímpar, a posição

\footnotetext{
8 "If the given number is odd, place a counter in position a, subtract one, and divide the remainder by two. Otherwise, if the given number is even, no counter is to be placed in position a. Always dividing by two and if the result is odd, subtracting one and placing a counter in that location, but if the result is even, placing nothing, until the given number is reduced to unity, for which a counter is placed in the position reached." (NAPIER, 2017, p.729)
} 
referente a ele será a de ordem um, logo a posição $a$ deve ser marcada (Figura 3), por outro lado, se o quociente da divisão do número dado for um valor ímpar, a posição referente a ele será a de ordem dois, pois ele será o segundo número a ser trabalhado, portanto a sua posição será $b$, que também deve ser indicada (Figura 3).

Figura 3 - Esquematização do processo de conversão por divisão com números ímpares

\begin{tabular}{|c|c|c|}
\hline $\mathbf{k}$ & 512 & \\
\hline $\mathbf{i}$ & 256 & \\
\hline $\mathbf{h}$ & 128 & \\
\hline $\mathbf{g}$ & 64 & \\
\hline $\mathbf{f}$ & 32 & \\
\hline $\mathbf{e}$ & 16 & \\
\hline $\mathbf{d}$ & 8 & \\
\hline $\mathbf{c}$ & 4 & \\
\hline $\mathbf{b}$ & 2 & \\
\hline a & 1 & $\mathbf{x}-1$ \\
\hline
\end{tabular}

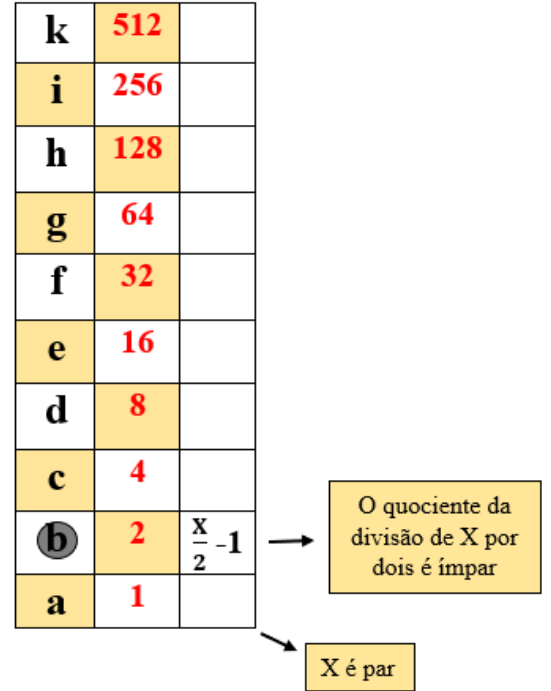

Fonte: Elaborado pelas autoras.

No entanto, caso o número dado "X”, ou os quocientes das divisões, sejam pares, as posições das ordens referentes a eles não devem ser marcadas. Logo, se o número fornecido for par, a posição relativa a ele, no caso a primeira, não deve ser indicada, da mesma forma com os restantes dos números trabalhados (Figura 4). 
Figura 4 - Esquematização do processo de conversão por divisão com números pares

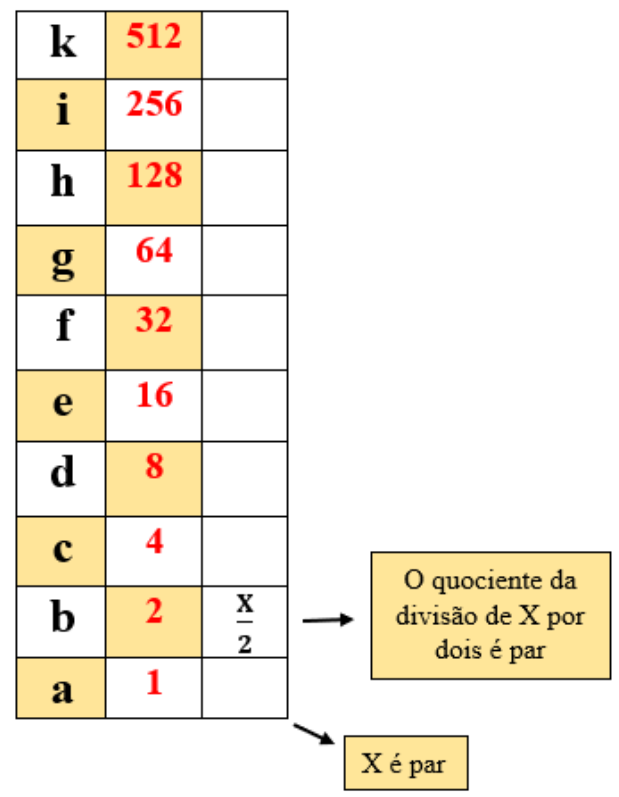

Fonte: Elaborado pelas autoras.

A título de exemplo, iremos converter novamente o número 20, agora utilizando o método de divisão por dois.

Figura 5 - Aritmética Linear na representação do método de conversão por divisão.

\begin{tabular}{|c|c|c|}
\hline $\mathbf{k}$ & 512 & \\
\hline $\mathbf{i}$ & 256 & \\
\hline $\mathbf{h}$ & 128 & \\
\hline $\mathbf{g}$ & 64 & \\
\hline $\mathbf{f}$ & 32 & \\
\hline e & 16 & $\mathbf{1 ( e )}$ \\
\hline d & 8 & 2 \\
\hline C & 4 & $5(c)$ \\
\hline $\mathbf{b}$ & 2 & 10 \\
\hline $\mathbf{a}$ & 1 & 20 \\
\hline
\end{tabular}$\quad$\begin{tabular}{|c|} 
impar \\
\hline
\end{tabular}

Fonte: Elaborado pelas autoras.

Primeiramente precisa-se indicar a paridade do valor dado, que no caso será par, com isso saberemos se há a necessidade de se indicar a posição referente a ele ou não. Como nosso número é par, deixa-se a posição $a$ em branco e dividiremos o valor por dois, resultando em 10. Da mesma forma encontra-se um outro número par, logo a posição 
referente a ele, a saber a posição $b$ por ser o segundo número trabalhado, também ficará em branco, ou seja, sem peças.

Em seguida divida 10 por dois, isso dá 5, portanto subtraia um e coloque uma peça na posição $c$, já que o número 5 é o terceiro valor encontrado. Após subtrair um, teremos o valor 4, dividindo-o novamente, resultaremos em 2, que por ser um número par, não precisará de peça na sua posição, isto é, a posição d. Por último, dividiremos o número 2, que resultará em um valor ímpar, pelo qual iremos demarcar a posição $e$. Assim, tem-se reduzido o número dado à unidade, finalizando o processo, conforme a Figura 5.

Agora, basta observar novamente as letras correspondentes às posições demarcadas (Figura 5) para escrever o número dado em notação local. Como utilizamos o mesmo número para ambos os processos de conversão a sua notação é a mesma, logo o número 20 pode ser escrito como ec, de acordo com a Figura 2 e 5.

Um número em sua forma $\operatorname{local}^{9}$ é importante para realizar operações utilizando a Aritmética de Localização, porém, ao finalizar a operação desejada, precisa-se convertêlo novamente, mas agora para sua forma comum ${ }^{10}$.

\section{Conversão de números locais para números comuns}

A mudança de um número local para um número comum também é realizada de duas formas, "por adição e por duplicação"11 (NAPIER, 2017, p.730, tradução nossa). Com base nisso, será abordado como se dá os processos presentes em cada um dos métodos propostos.

Segundo Almeida e Pereira (2020, p.46), o método por adição "significa reunir os números locais nas posições marcadas e somar os valores correspondentes a eles". Isto é, ao pegar um número em sua forma local, basta somar os valores de suas representações para encontrar o número em sua forma comum. Exemplificando, dado um número cuja sua notação local é ef, precisamos saber que valores representa cada uma das letras contidas nessa notação. Nesse caso, tem-se a letra $e$ que corresponde ao valor 16 e a letra $f$ que corresponde ao valor 32 , logo, ao somar esses valores ${ }^{12}$ acha-se o número 48 , na sua forma comum (Figura 6).

\footnotetext{
${ }^{9}$ Ou seja, escrito na forma alfabética.

${ }^{10}$ Isto é, escrito na forma usual, ou números naturais.

11 "By addition and by doubling" (NAPIER, 2017, p.730)

$1216+32$
} 
Figura 6 - Aritmética Linear na representação do método de conversão por adição.

\begin{tabular}{|c|c|c|}
\hline $\mathbf{k}$ & $\mathbf{5 1 2}$ & \\
\hline $\mathbf{i}$ & 256 & \\
\hline $\mathbf{h}$ & 128 & \\
\hline $\mathbf{g}$ & 64 & \\
\hline $\mathbf{f}$ & 32 & $\mathbf{3 2}$ \\
\hline e & 16 & $\mathbf{1 6}$ \\
\hline $\mathbf{d}$ & $\mathbf{8}$ & \\
\hline $\mathbf{c}$ & 4 & \\
\hline $\mathbf{b}$ & 2 & \\
\hline a & 1 & \\
\hline
\end{tabular}

Fonte: Elaborado pelas autoras.

Quanto ao método de duplicação, Napier (2017, p.730, tradução nossa) explica que

\begin{abstract}
O método para retornar da notação local para a normal por duplicação é do seguinte modo. Para a posição que denota o último (ou maior) número, duplique uma unidade. A este adicione um se você encontrar uma peça na penúltima posição, mas se não, não acrescente nada. [...] Continue dobrando, somando uma unidade sempre que você encontrar uma peça, até chegar à primeira posição, marcada $a .{ }^{13}$
\end{abstract}

Desse modo, o método de duplicação também consiste em dois processos, que estão relacionados a como estão dispostas as peças na barra de Artimética Linear. A primeira duplicação a ser realizada é a de uma unidade para a posição mais alta representada na barra, não importando qual seja ela.

Após isso, a cada duplicação realizada observa-se se existem peças na próxima posição, caso haja, deve-se somar uma unidade àquele número já duplicado. Por exemplo, se o número mais alto na atual notação local for o 64, que é apresentado na posição $g$, tem-se que duplicar uma unidade referente à ela, resultando em 2, em seguida será preciso observar se existe uma peça na próxima posição, a saber $f$, se houver, deve-se somar um ao número duplicado, que ficará 3 , e só depois realizar a próxima duplicação.

Todavia, caso não haja número na posição seguinte ao valor mais alto, não será preciso acrescentar nada, basta duplicá-lo novamente. Utilizando o exemplo anterior, se

\footnotetext{
13 "The method for returning from local to ordinary notation by doubling is as follows. For the position that denotes the last (or highest) number, double unity. To this add one if you find a counter in the penultimate position, but if not, add nothing [...] Keep on doubling, adding unity whenever you strike a counter, until you arrive at the first position, marked a" (NAPIER, 2017, p.730)
} 
a posição $f$, não estivesse representada com uma peça, o próximo número a ser duplicado seria o próprio 2, que é o valor da nossa primeira duplicação. Esses procedimentos são repetidos até chegar na primeira posição da haste, que será a posição $a$. Na ficura 7, temse uma ilustração da conversão do número local $f e$, agora utilizando o processo de duplicação.

Figura 7 - Artimética Linear na representação do método de conversão por duplicação.

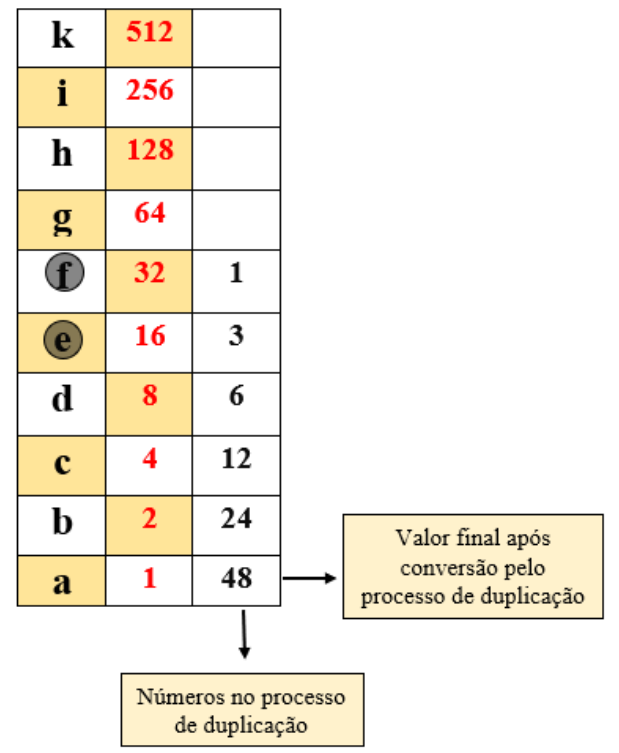

Fonte: Elaborado pelas autoras.

Inicialmente, é preciso dobrar o valor 1 para a posição mais alta da barra, que no caso será a posição $f$, resultando em 2 , em seguida observa-se que na posição anterior, isto é, a posição $e$, contém uma peça, logo, antes de realizar a segunda duplicação, devese acrescentar uma unidade ao nosso valor, o que dá 3. Em seguida dobra-se novamente e econtrando o valor 6 , como não há nenhuma peça na posição $d$, não será preciso acrescentar nada, e dobre novamente, o que dá 12, mas novamente não acrescente nada, pois a posição $c$ está vaga. Duplicando outra vez, o resultado será 24 , mas como não há peças na posição $b$, não precisará somar nada. Dobrando mais uma vez encontra-se o valor 48, chegado à primeira posição $a$ e finalizado o processo, pois não existe peças nessa posição. Finalmente, o número 48 que surge na posição $a$, é o número em sua forma comum (Figura 7).

Entender como funciona as conversões de um número comum para o local, e vice versa, é a base para realizar as operações utilizando o método de Aritmética de 
Localização. Porém existem outros procedimentos que são fundamentais durante as execuções desses cálculos, os quais serão tratados a seguir.

\section{Os conhecimentos matemáticos mobilizados no processo de conversão dos números de Napier}

De acordo com o que foi abordado a respeito da construção e conversões de números realizadas na Aritmética Linear, pode-se observar que diversos conhecimentos matemáticos são mobilizados. Se for realizada uma busca mais pontual, as conversões númericas é um assunto que está muito presente na área da computação, no qual são trabalhadas as bases e as representações. Uma das bases mais comuns é a binária, no qual consiste de um sistema de númeração em que os valores são denotados como uma soma de potências de base dois, muito parecido com a representação do número local de Napier.

Com isso, nota-se que o processo realizado com as barras de Aritmética Linear pode servir como um coadjutor na assimilação dessas conversões númericas, no que refere-se à base binária, pois o próprio método já atua com esse sistema.

Ainda a repeito das conversões realizadas pode-se verificar que, nos quatro processos discutidos, as operações de subtração e divisão estão inseridas na mudança de um número local para o comum e as operações de adição e multiplicação são exibidas na transformação de um número comum para o local. Quanto à importância desse conhecimento, a Base Nacional Comum Curricular (BNCC) informa que,

\footnotetext{
No processo da construção da noção de número, os alunos precisam desenvolver, entre outras, as ideias de aproximação, proporcionalidade, equivalência e ordem, noções fundamentais da Matemática. A proporcionalidade, por exemplo, deve estar presente no estudo de operações com os números naturais. (BRASIL, 2018, p.268).
}

Dessa forma, considera-se que o uso desse dispositivo auxilie no ensino das operações básicas de aritméticas, facilitando a compreensão de cálculos considerados dificeis para alunos do ensino fundamental e médio, em particular no estudo da divisão. Além disso, a barra de Aritmética Linear possibilita o estudo de Progressão Geométrica (PG), visto que os valores inscritos na haste são uma sequência númerica em que cada termo é igual ao produto do termo anterior por uma constante, que no caso será de razão igual a 2 . 
Esse conhecimento é apresentado na BNCC como uma habilidade em que é preciso “identificar e associar progressões geométricas (PG) a funções exponenciais de domínios discretos, para análise de propriedades, dedução de algumas fórmulas e resolução de problemas" (BRASIL, 2018, p.541). Portanto existe um potencial didático também no processo de construção da barra, podendo ser direcionado para o ensino desse conteúdo no Ensino Médio.

\section{Algumas considerações}

A importância do papel da história da matemática no ensino é desenvolver atividades que "focalizassem não só conteúdo e método, mas também os critérios e as razões, devidamente contextualizados, do processo da elaboração do conhecimento" (PEREIRA E SAITO, 2019, p.345). Uma das formas de fazer isso é através dos instrumentos matemáticos, visto que expressam conceitos matemáticos antigos que podem auxiliar-nos no processo de entendimento de como o conhecimento era retratado em uma determinada época e como podemos assimilá-lo atualmente com um tratamento didático.

Nessa perspectiva a utilização da história da matemática no ensino pode ajudar a formar alunos a contextualizar os conhecimentos e os inserir numa perspectiva de construção humana e coletiva, de acordo com Guimarães e Marinheiro (2017).

Nesse estudo, ainda inicial, o processo de conversão dos números locais e comuns na Aritmética Linear, presente no método de Aritmética de Localização de John Napier, pode incorporar diferentes conhecimentos aritméticos com um direcionamento para as operações matemáticas, como adição, subtração, divisão e multiplicação, além de progressões geométricas (PG) e números binários. Entretanto, é necessário que haja uma aplicação tanto na formação de professores quanto na Educação Básica para validar possíveis intercorrências didáticas e pedagógicas no processo de ensino e aprendizagem desses níveis.

\section{Referências}

ALBUQUERQUE, L.; GONTIJO, C. A complexidade da formação do professor de matemática e suas implicações para a prática docente. Revista Espaço Pedagógico, v. 20, n. 1,4 out. 2013. 
ALMEIDA, J. P. D.; PEREIRA, A. C. C. A aritmética de localização de John Napier para a multiplicação. Revista história da matemática para professores, v. 6, n. 2, p. 43 - 56, 31 dez. 2020. Disponível em:

http://www.rhmp.com.br/index/index.php/rhmp/article/view/115. Acesso em: $1 \mathrm{de}$ fev. de 2021.

BRASIL. Ministério da Educação. Base Nacional Comum Curricular. Brasília, 2018.

GUIMARÃES, Ueudison Alves; MARINHEIRO, Carlos Alberto. A História da

Matemática no Ensino Fundamental. Revista Científica Multidisciplinar Núcleo do

Conhecimento. Ano 02, Ed. 01, Vol. 16, pp. 05-11, março de 2017. ISSN: 2448-0959

NAPIER, John. Rabdologiae, Seu Numerationis Per Virgulas: cum appendice de expeditíssimo Multiplicationes promptuario, quibus acessit e arithmeticea localis liber unus. In: RICE, B.; GONZÁLEZ-VELASCO, E.; CORRIGAN, A. The Life and Works of John Napier. Cham: Springer, 2017. p. 652-749.

PEREIRA, Ana Carolina Costa; SAITO, Fumikazu. Os instrumentos matemáticos na interface entre história e ensino de matemática: compreendendo o cenário nacional nos últimos 10 anos. Boletim Cearense de Educação e História da Matemática, v. 5, n. 14, p. 109-122, 2018. Disponível em:

https://revistas.uece.br/index.php/BOCEHM/article/view/225/174. Acesso em: $1 \mathrm{fev}$. 2021.

PEREIRA, Ana Carolina Costa; SAITO, Fumikazu. A reconstrução do báculo de Petrus Ramus na interface entre história e ensino de matemática. Revista Cocar, v. 13, n. 25, p. 342-372, 2019.

PRODANOV, Cleber Cristiano; FREITAS, Ernani César de. Metodologia do Trabalho Científico: Métodos e Técnicas da Pesquisa e do Trabalho Acadêmico. 2. ed. Novo Hamburgo: Editora Feevale, 2013. 277 p.

SAITO, Fumikazu. DIAS, Marisa da Silva. Interface entre história da matemática e ensino: uma atividade desenvolvida com base num documento do século XVI. Ciência \& Educação, Bauru, v. 19, n. 1, p.89- 111, mar. 2013. Quadrimestral.

SAITO, Fumikazu. Construindo Interfaces Entre História e Ensino da

Matemática. Ensino da Matemática em Debate, São Paulo, v. 3, n. 1, p. 3-19, 2016.

Disponível em: https://revistas.pucsp.br/index.php/emd/article/view/29002. Acesso em: 1 fev. 2021.

Recebido em: 25 / 02 / 2021

Aprovado em: 14 / 04 /2021 